\title{
THE BRAIN TARGETED DRUG DELIVERY SYSTEM
}

\author{
Priyanka A. Bire ${ }^{1 *}$, Harsha V. Sonaye ${ }^{2}$, Dr. C.A. Doifode ${ }^{3}$, Preetesh C. Khekare ${ }^{4}$
}

*1Plot no.32, Chandrikapure layout, Nirmal Colony Road, Nara Road, Nagpur, 440014, Maharashtra Shri Sacchidanand Shikshan Santha's College of Pharmacy, Koradi Contact No: +917387994780 E-mail id:

$$
\text { pbire7@gmail.com }
$$

${ }^{2}$ At-29, Shrinagar layout, Batchalar road, Wardha, 441107, Maharashtra Shri Sacchidanand Shikshan Santha's College of Pharmacy, Koradi Contact No: +919970794970E-mail id: harsha_20054@rediffmail.com

343-A, Ram nagar, Nagpur, 440010, Maharshtra, Shri Sacchidanand Shikshan Santha's College of Pharmacy, Koradi Contact No: +91926074273 E-mail id: chandu581@rediffmail.com

${ }^{4}$ At- Chichapura ward no.9, Saoner, Dist-Nagpur, 441107, Maharashtra, Shri Sacchidanand Shikshan Santha's

College of Pharmacy, Koradi Contact No: +917776000580,8857945877E-mail id: khekarepr@gmail.com

\section{*Corresponding Author: -}

E-mail id: pbire7@gmail.com

\begin{abstract}
: -
The brain is a delicate organ, and nature has very efficiently protected it. The drug accessibility to the central nervous system $(C N S)$ is limited by the blood brain barrier (BBB). Various brain disorders are Parkinson, Alzheimer, Meningitis, Brain abscess, Epilepsy, Multiple sclerosis, Late-stage neurological trypanosomiasis (Sleeping sickness) etc. The management of brain related diseases with the present available therapeutic system is very difficult, as insufficient amount of drug reaches to the brain, due to highly lipophilic nature of blood-brain barrier (BBB). Drug delivery to the brain requires advances in drug delivery technologies. Due to the presence of the blood brain barrier only small lipid soluble drug in the circulation are delivered to the brain cells. Therefore, practical strategies are required for mediating the drug transport across blood brain barrier.
\end{abstract}

Keywords: Brain, Barriers, Approaches, Strategies to CNS Drug delivery, Recent Advances in Brain Targeting.

\section{(c) $(\$)$}




\section{INTRODUCTION}

The brain is important organ, it is protected with Blood Brain Barrier and it is very difficult to target it, unless using modified drug delivery system. The blood-brain barrier is made up of endothelial cells, which are connected by tight junctions. The blood-brain barrier only receives the material like water, some gases, and lipid-soluble molecules by passive diffusion method, also the selective transport of molecules such as glucose and amino acids. Astrocytes are formed blood-brain barrier. BBB is one such barrier which separates but not isolates brain from all other body components. ${ }^{1-3}$ The decades of research in the development of drugs delivery for brain disease, crossing of blood brain barrier is a key obstacle. So the BBB is a life saving supporting media that protect brain against the toxic substance that circulate in the blood stream, its existence is a severe limitation to the delivery of most of the drugs to the brain because they do not cross the BBB in sufficient amounts. Many serious CNS disorders of the brain such as Alzheimer disease, stroke/neuro protection, brain and spinal cord injury, brain cancer, HIV infection of the brain, amyotrophic lateral sclerosis (ALS), Huntington disease and childhood inborn genetic errors affecting the brain, Parkinson disease and multiple sclerosis (MS), to reach the therapeutic drug level in the brain.

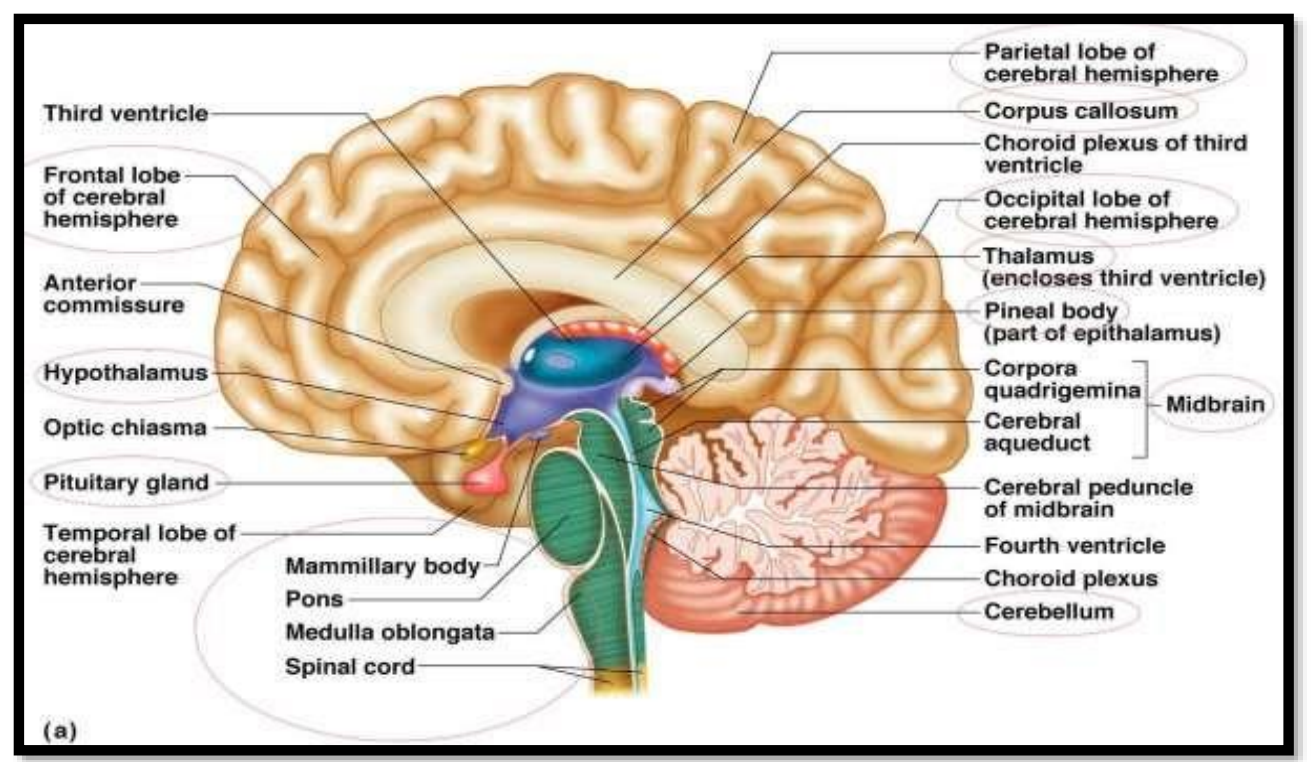

Fig.1: Figure showing Areas of Brain

A novel delivery system such as Nanoparticulate system as drug carriers with high loading capacity and small particle size, which bypass the Reticule endothelial system (RES), are considered as suitable delivery system ${ }^{4}$. To study BBB have provides new opportunities for increasing drug delivery to the CNS. Different techniques are used for targeting the blood brain barrier for drug delivery to the brain include carrier system, prodrug approach, liposomes, nanoparticles, dendrimers.

\section{Blood-Brain Barrier (BBB) 2. Blood-Cerebrospinal Fluid Barrier (BCF) 3. Blood-Tumor Barrier ${ }^{1-3}$ 1. Blood Brain Barrier (BBB)}

Basal membrane and brain cells, such as pericytes and astrocytes, surrounding the endothelial cells further form and maintain an enzymatic and physical barrier known as the blood-brain barrier (BBB). BBB tight junctions are formed between endothelial cells in brain capillaries, thus preventing paracellular transport of molecules into the brain.

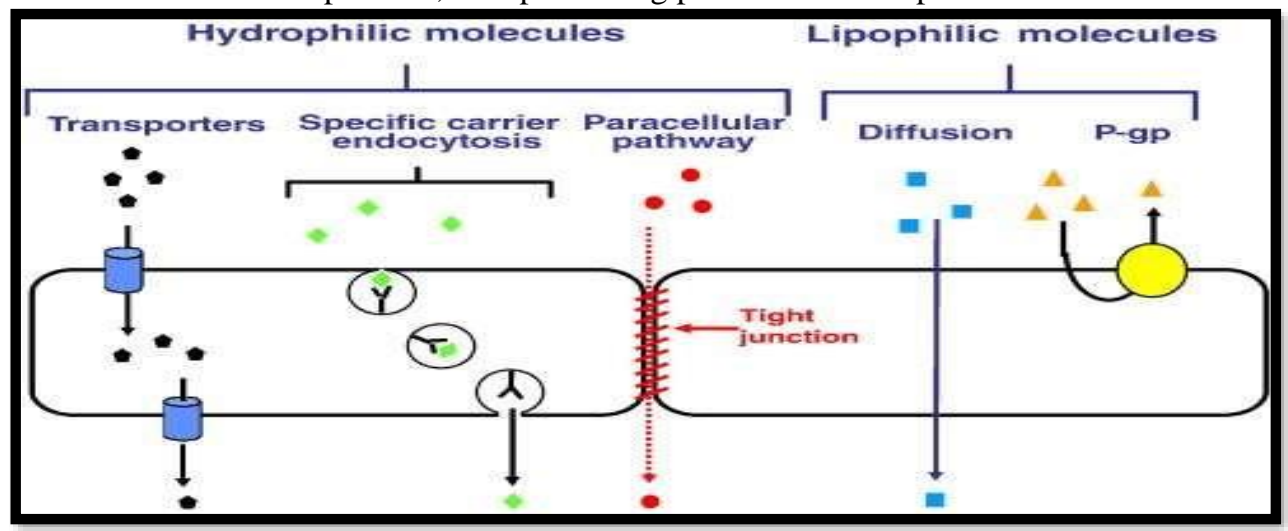

Fig. 2: Schematic representation of the transport of molecules across the BBB

Micro-vessels small in diameter and thin walls compared to vessels in other organs make up an estimated $95 \%$ of the total surface area of the BBB, and represent the principal route by which chemicals enter the brain. In brain capillaries, intercellular cleft, pinocytosis, and fenestrate are virtually nonexistent; exchange must pass transcellularly. Therefore, 
only lipid- soluble solutes that can freely diffuse through the capillary endothelial membrane may passively cross the BBB. ${ }^{5}$

\section{Advantages}

1. Side effect and toxicity reduces.

2. Dose of drug reduces by targeting organ.

3. Avoids degradation of drug (first pass metabolism).

4. Bioavailability increases.

5. Fluctuation in concentration decreases.

6. Permeability of proteins and peptide increases. ${ }^{6,7}$

\section{Blood-Cerebrospinal Fluid Barrier (BCSFB)}

Another barrier between the blood and the brain is the blood-cerebrospinal fluid barrier (BCSFB), which separates the blood from cerebrospinal fluid (CSF). However, this barrier is not considered as a main route for the uptake of drugs since its surface area is 5000-fold smaller than that of the BBB. ${ }^{8-10} \mathrm{CSF}$ can exchange molecules with the interstitial fluid of the brain parenchyma; the passage of blood-borne molecules into the CSF is also carefully regulated by the BCB. Physiologically, the BCB is found in the epithelium of the choroids plexus, which is arranged in a manner that limits the passage of molecules and cells into the CSF. ${ }^{11-13}$ The choroid plexus and the arachnoid membrane act together at the barriers between the blood and CSF. ${ }^{14}$ The arachnoid membrane is generally impermeable to hydrophilic substances, and its role is formation of the Blood-CSF barrier, is largely passive. The choroid plexus forms the CSF and actively regulates the concentration of molecules in the CSF.

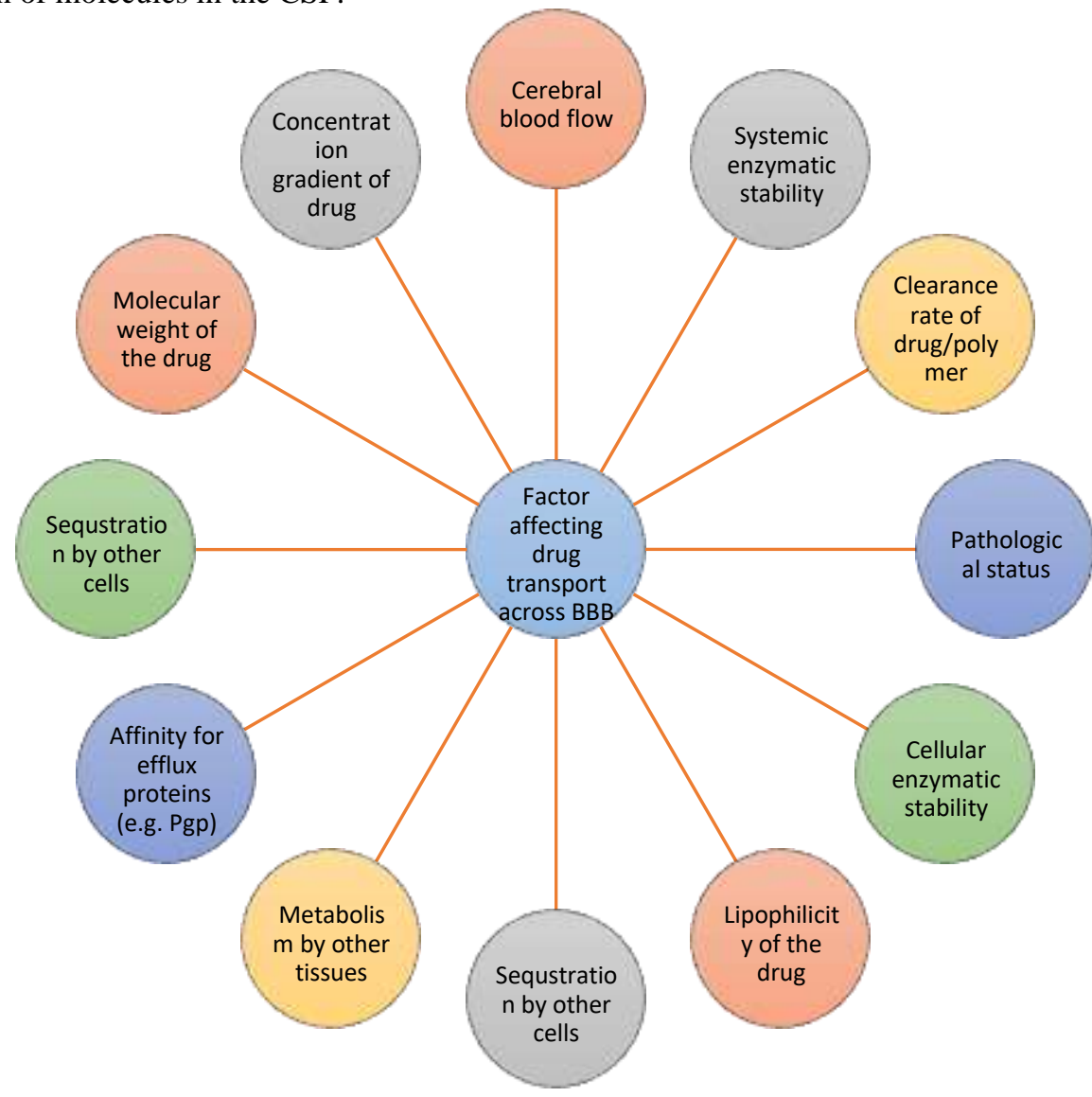

Fig. 3: Schematic representation of the factors affecting drug transport across the BBB

\section{Blood-Tumor Barrier}

Intracranial drug delivery becomes even more challenging when the target is a CNS tumor.

The presence of the BBB in the microvasculature of CNS tumors has clinical consequences ${ }^{15}$. In CNS malignancies where the BBB is significantly compromised, a variety of physiological barriers common to all the solid tumors inhibit drug delivery via the cardiovascular system. Drug delivery to neoplastic cells in a solid tumor is compromised by a heterogeneous distribution of microvasculature throughout the tumor interstitial, which leads to spatially inconsistent drug delivery. However, as a tumor grows large, the vascular surface area decreases, leading to reduction in trans-vascular exchange of blood-borne molecules. At the same time, intra-capillary distance increases, leading to a greater diffusional requirement for drug delivery to neoplastic cells and due to high interstitial tumor pressure and the associated peri-tumoral edema leads to increase in hydrostatic pressure in the normal brain parenchyma adjacent to the tumor. As a result, the cerebral microvasculature in these tumor adjacent regions of normal brain may be even less permeable to drugs than 
normal brain endothelium, which leads to exceptionally low extra-tumoral interstitial drug concentrations ${ }^{16}$. Brain tumors may also disrupt BBB, but these are also local and non-homogeneous disruptions ${ }^{17}$.

\section{APPROACHES TO CNS DRUG DELIVERY}

Basically, two methods have been described in the literature to actively enhance drug delivery to the brain after systemic administration: either opening/disruption of the neuroprotective BBB by osmotic imbalance, ultrasound or vasoactive compounds (e.g., bradykinin or P-glycoprotein inhibitors), or physiological strategies aiming to use endogenous transport mechanisms. While the first method has the disadvantage that those neurons may be damaged (semi)- permanently due to unwanted blood components entering the brain ${ }^{18-22}$. The physiological strategies have a large potential as discussed in several review papers elsewhere ${ }^{23}$. As a third alternative (using a combination of aspects of both methods), positive charge has also been applied to compounds or drug carriers to quite effectively enhance the absorptive- mediated transport across the $\mathrm{BBB}^{24-25}$, however, a beneficial therapeutic window of this basically toxic transport mechanism has thus far not been established. To overcome the multitude of barriers restricting CNS drug delivery of potential therapeutic agents, numerous drug delivery strategies have been developed. These strategies generally fall into one or more of the following categories: invasive, non- invasive or miscellaneous techniques ${ }^{26-28}$. The CNS drug delivery tree encompassing the various possible strategies is shown below in the (Fig.3)

\section{A. Invasive}

- Intracerebroventricular (ICV) infusion

- Convection-enhanced delivery (CED)

- Intra-cerebral injection or implants

- Disruption of the BBB.

\section{B. Non-invasive}

- Chemical techniques

a. Prodrug

- Colloidal Techniques

a. Nanoparticles

b. Liposomes

\section{Miscellaneous techniques}

a. Intranasal delivery

b. Iontophoretic delivery



Fig. 4: Schematic representation of the different drug delivery approaches to the CNS

BRAIN DRUG DELIVERY STRATEGIES 1. Conventional strategies

a) Neurosurgical or invasive strategies

Hyperosmolar BBB disruption

Intraventricular drug infusion 
Intracerebral implants

b) Physiologic-based strategies:

Pseudo-nutrients

Cationic antibodies

Chimeric peptides

c) Pharmacological strategies:

Chemical delivery systems

Liposomes

Nanoparticles

\section{Recent advances}

a) Dendrimers

b) Polyanhydrides

c) Lipoplexes and Polyplexes

d) Scaffolds

e) Convection-enhanced delivery

f) Modified nanoparticles:

Multifunctional nanoparticles Magnetic nanoparticles ${ }^{29}$

\section{PHARMACEUTICAL TECHNOLOGY-BASED STRATEGIES}

- The technological strategies are essentially non-invasive methods of drug delivery to the CNS and represent valuable approaches for enhancing transcellular permeability of therapeutic agents and biomacromolecules across the BBB.

- They are based on the use of nanosystems (colloidal carriers), mainly liposomes and polymeric nanoparticles even though other systems such as solid lipid nanoparticles, polymeric micelles and dendrimers are also being tried.

- An important requirement of the systemic intravenous use of these nanocarriers is their ability to circulate in the bloodstream for a prolonged period of time.

- However, after intravenous administration, they interact with the reticuloendothelial system (RES) which removes them from the blood stream.

- This process mainly depends on particle size, charge and surface properties of the nanocarrier ${ }^{30-31}$. To prevent the uptake by the RES, poly ethylene glycol (PEG) coating or direct chemical linking of PEG to the particle surface provides relatively long plasma residence times. In fact, these nano carriers can be taken up actively by carriermediated transport (CMT), receptor- mediated endocytosis (RME) and adsorptive-endocytosis (AME) and hence reaches the cerebral parenchyma, or is degraded within lysosomes leading to the drug being released into the brain tissues.

\section{Liposomes}

- Liposomes are small vesicles (usually submicron-sized) comprising of one or more concentric bilayers of phospholipids separated by aqueous compartments.

- It has also been suggested that liposomes could enhance drug delivery to the brain across the BBB.

- Although liposomes have been reported to enhance the uptake of certain drugs into the brain after intravenous injection.

- Liposomes are sterically stabilized by attaching ligands to the surface of the liposomes ${ }^{33-34}$. A recent application of transferrin surface- conjugated liposomes includes the delivery of the anticancer drug 5-fluorouracil (5-FU) to brain.

- 5-FU is one of the most powerful anticancer agents, but cannot reach an effective concentration in the brain tumor cells when administered systemically.

- Modified liposomes have also been used for enhanced gene delivery to brain tumors. 


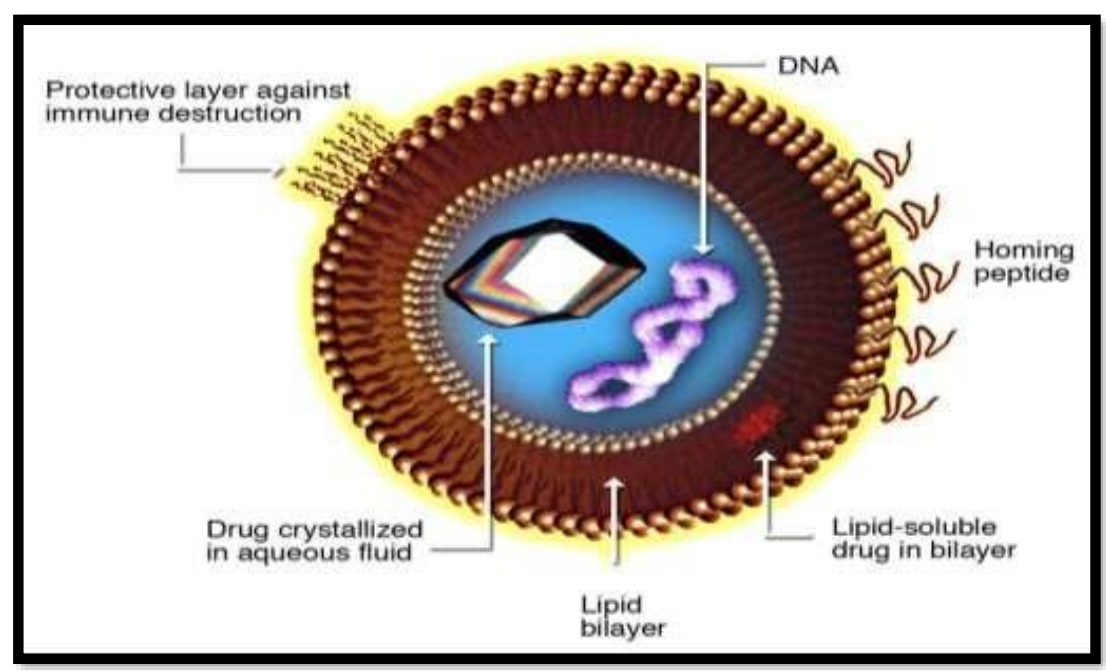

\section{Advantages}

Fig.5 Liposomes for Drug Delivery

- Liposomes supply both a lipophilic environment and aqueous "milieu interne" in one system and are therefore suitable for delivery of hydrophobic, amphipathic and hydrophilic drugs and agents.

- Liposomes could encapsulate not only small molecules but also macromolecules like superoxide dismutase, haemoglobin, erythropoietin, interleukin-2 and interferon-g.

- Liposomes reduced toxicity and increased stability of entrapped drug via encapsulation (eg.Amphotericin B, Taxol).

- Liposomes help to reduce exposure of sensitive tissues to toxic drugs.

- Alter the pharmacokinetic and pharmacodynamic property of drugs (reduced elimination, increased circulation life time).

\section{Limitation}

- High production cost

- Leakage and fusion of encapsulated drug / molecules.

- Sometimes phospholipid undergoes oxidation and hydrolysis

- Short half-life

- Low solubility

- Less stability

\section{Nanoparticles}

- Nanoparticles (NPs) are solid colloidal particles made up of polymeric materials ranging in size from 1-1000 nm. This definition includes both nanocapsules, with a core-shell structure (a reservoir system), and nanospheres (a matrix system).

- NPs are used as a carrier system in which the drug is dissolved, entrapped, encapsulated, adsorbed or chemically linked to the surface ${ }^{35}$. NPs possess the advantage of a high drug loading capacity and can provide protection against chemical and enzymatic degradation. Examples of synthetic polymers used to prepare NPs are poly (alkylcyanoacrylate) (PACA), acrylic copolymers, poly(D,L-lactide-co-glycolide), and poly(lactide).

- NPs have also been prepared from natural proteins (albumin and gelatin) and polysaccharides, starch and chitosan). Like liposomes, NPs are rapidly cleared from the blood following intravenous administration.

- As carriers for drug delivery to the brain, NPs need to be small $(<100 \mathrm{~nm})$ to avoid the RES, neutrophil activation, platelet aggregation, and inflammation.

- Besides solid polymeric nanoparticles (NPs), solid lipid nanoparticles (SLN) have also been employed for delivering drugs to the CNS. SLN are dispersions of solid lipids stabilized with emulsifier or emulsifier/co-emulsifier complex in water.

- In particular, delivery to the brain of antitumor drugs, including camptothecin, doxorubicin and paclitaxel, incorporated into SLNs and PEGylated SLNs is studied ${ }^{[36-37]}$. In all of the studies it has been found that significantly higher drug concentrations is detected in the brain when the antitumor drug is encapsulated and delivered in a SLN.

- It is therefore a noteworthy finding that SLNs appear by their nature to be capable of overcoming the BBB.In comparison with surfactant coated polymeric NPs (specifically useful in bypassing BBB), SLN have also been evaluated for brain delivery of the potent and frequently used HIV protease inhibitor (PI), atazanavir, that, like other PIs exhibits low brain permeability ${ }^{38}$.

\section{Polymeric Micelles and Microemulsions}

- Polymeric micelles as drug delivery systems are formed by amphiphilic copolymers having an A-B dib lock structure with A, the hydrophilic (shell) and B, the hydrophobic (core) polymers. The polymeric micelles are thermodynamically and kinetically stable in aqueous media. The size of polymeric micelles usually varies from ca. 10 to $100 \mathrm{~nm}$. 
- This narrow size range is similar to that of viruses and lipoproteins. The core is composed of hydrophobic polymer blocks [e.g., poly (propylene glycol) (PPG), poly (D, L-lactide), poly (caprolactone), etc.] and a shell of hydrophilic polymer blocks (e.g., PEG). Most of them are biodegradable and biocompatible.

- Earlier studies have shown that poloxamer (PluronicTM) micelles conjugated with antibodies may improve brain distribution of haloperidol, a neuroleptic agent. This approach resulted in a dramatic improvement of drug efficacy. This result indicates that Bio-conjugates, Biomimetic polymers provide an effective transport of solubilized neuroleptic agents across the $\mathrm{BBB}^{39-41}$.

\section{Dendrimers}

- Dendrimer is a highly branched polymer molecule formed by a central core to which the branches are attached, the shell of the branches surrounding the core, and the surface formed by the branch's termini.

- They are of small size comparable to that of polymeric micelles or nanoparticles of small dimensions. Thus, for instance, a typical dendrimer molecule, such as poly (amidoamine) (PAMAM) dendrimer, has a diameter ranging from 1.5 to $14.5 \mathrm{~nm}$.

- As carriers for drug delivery to the brain, dendrimers conjugates with anti-cancer agents have been studied for the treatment of tumors at CNS level.

- In addition, gene delivery into brain has been also shown using a transferring conjugated PEGmodified PAMAM dendrimer ${ }^{42}$.

\section{Peptide-Vector-Mediated Strategy}

- The other approach for the delivery of neuropharmaceuticals is the use of small naturally derived peptides that cross cellular membranes efficiently, for example, pegelin and penetratin peptides (18 and 16 amino acids, respectively).

- SynB1 and pegelin (RGGRLSYSRRRFSTSTGR; molecular mass 2099 Da) is derived from natural peptides called protegrins.

- They have an amphipathic structure in which the positively charged and hydrophobic residues are separated in the sequence.

- Replacement of the four cysteine residues with serine residues leads to linear peptides (pegelin). The potential of this approach as an effective delivery system for transporting drugs across the BBB has been demonstrated in animal models ${ }^{43}$.

\section{Novel Methods}

- The challenging domain of effective brain delivery has led to a keen scientific pursuit and as a result many novel methods have been invented and patented.

- In these series, researchers have revealed the use of iontophoresis as an adjuvant for CNS drug delivery.

- Iontophoresis has been defined as the active introduction of ionised molecules into tissues by means of an electric current.

- The parent US patent method and device for delivery of a biologically active agent that is transported by means of iontophoresis and/or phonophoresis directly to the CNS using the olfactory pathway to the brain and thereby circumventing the BBB and is known as transnasal iontophoretic delivery ${ }^{44}$.

\section{RECENT ADVANCES IN BRAIN TARGETING Polyanhydrides}

Polyanhydrides are bio-degradable polymers which release the drug mainly by simple hydrolysis. Polyanhydrides are basically intracerebral implants useful for controlled drug delivery. In Polyanhydrides, drug is embedded in polymer matrix $^{45}$. They degrade uniformly into non-toxic metabolites that are non- inflammatory, non-mutagenic and noncytotoxic. Their degradation profile can be modulated from days to months by varying the type and ratio of the monomers. Polyanhydrides are believed to predominantly undergo surface erosion due to:

The high hydrophilicity of the anhydride bonds on the surface Hydrophobicity, which restricts water penetration into the bulk

A decrease in the device thickness throughout the erosion process, maintenance of the structural integrity and the nearly zero-order degradation kinetics suggest that the heterogeneous surface erosion is taking place. High hydrolytic reactivity of the anhydride linkage provides versatility and control of degradation ${ }^{46}$. The drug is slowly released from these for three weeks to destroy any remnants of tumor. Because of the local delivery of the drug, harmful systemic side effects are avoided $^{47}$.

\section{Polyanhydrides in brain tumor}

- Brain tumor Glioblastoma multiforme (GBM) accounts for about $80 \%$ of adult primary brain tumors and are usually found in the cerebral hemispheres.

- Many of the anti-cancer drugs have large molecular structures, ionic charge or are hydrophilic and thus are unable to cross the BBB, and intolerably high systemic levels are required to achieve the therapeutic doses within the CNS. 


\section{Lipoplxes and Polyplexes}

- In order to improve the delivery of the new DNA into the cell, the DNA must be protected from damage and its entry into the cell must be facilitated. Lipoplexes and polyplexes serve this purpose.

- Both have the ability to protect the DNA from undesirable degradation during the transfection process. Plasmid DNA can be covered with lipids in an organized structure like a micelle or a liposome. When the organized structure is complexed with DNA it is called a lipoplex. There are three types of lipids Anionic (negatively charged) Neutral Cationic (positively charged)

- Initially, anionic and neutral lipids were used for production of lipoplexes for synthetic vectors. Lipoplexes are compatible with body fluids, there is a possibility of adapting them to be tissue specific and there is very little toxicity associated with them. The only disadvantage is that they are complicated and time consuming to produce.

- Thus, attention was turned to the cationic versions. Cationic lipids, because of the positive charge on them, have ability to naturally complex with the negatively charged DNA. Also because of the positive charge, they interact with cell membrane, endocytosis of the lipoplex occurs and the DNA is released into the cytoplasm.

- The cationic lipids also protect against degradation of the DNA by the cell. Cationic lipids include Dioleoyl phosphoethanolamine (DOPE), dioleyloxy trimethylammonium chloride (DOTMA) ${ }^{49}$.

- Sawant et al. formulated TATp (TAT plasmid) lipoplexes with cationic lipid N- [1-(2,3Dioleoyloxy) propyl]-N, N, Ntrimethylammonium methyl sulfate (DOTAP). The lipoplexes showed higher transfection efficiency in vitro when incubated with mouse fibroblast ${ }^{50}$.

- Complexes of polymers with DNA are called polyplexes. Most polyplexes consist of cationic polymers and they are formed by ionic interactions. One large difference between the methods of action of lipoplexes and polyplexes is that polyplexes cannot release the associated DNA into the cytoplasm.

- For release to take place cotransfection with endosomelytic agents which lyse the endosome must occur and the polyplex enters the cell. However, this is not always the case, polymers such as Polyethylenimine (PEI), chitosan and trimethyl chitosan have their own method of endosome disruption. PEI forms dense nanosized particulates and complexes with negatively charged DNA by electrostatic interactions.

- The PEI/DNA complex takes overall positive charge and interacts with negatively charged components of cell membranes and enter cells by endocytosis. The PEI/DNA complex enters the cells by non-specific adsorption mediated endocytosis. Upon endocytosis, PEI undergoes further protonation as the endosomal compartment acidifies.

- Protonation of PEI occurs by capturing protons, which is called as Proton Sponge mechanism. This leads to osmotic swelling and subsequent endosomal disruption. Zhanta et al. demonstrated that only one nuclear localization signal (NLS) peptide covalently linked to DNA could increase the transfection efficacy following an intracellular injection of the plasmid ${ }^{51}$.

\section{Scaffolds}

- Scaffolds are implantable and can be used to treat a variety of conditions associated with brain injury and diseases, for delivering drugs to treat neurological diseases such as Parkinson's disease and Alzheimer's disease.

- Delivery of therapeutic agents from scaffolds potentially helps to limit the damage to neurons while helping to preserve their function.

- Although scaffolds have wide range of potential applications for neural tissue engineering, the brain presents similar obstacles when designing scaffolds. Considerations include Minimizing cell death and inflammation after implantation of scaffolds, by choosing biocompatible materials

Controlling drug release over an appropriate time period to prevent multiple surgeries or injections

Making the whole process minimally invasive to preserve the integrity of the BBB Scaffolds should be small and minimally invasive

\section{Scaffolds for brain drug delivery}

Woerly $\mathrm{S}$ et al. studied efficacy of poly (hydroxyl phenyl mathacrylate) [PHPMA] and PHEMA scaffolds containing glucosamine or $\mathrm{N}$-acetylglucosamine groups when implanted between the septum and the hippocampus in a fimbria-fornix lesion cavity. It was found that PHEMA scaffolds showed markedly less infiltration of connective tissue than PHPMA ${ }^{52}$.

\section{Convection-enhanced delivery}

- Intraoperative injection of anti-cancer agents into brain tumors has limited success due to diffusion-limited drug distribution as well as leakage away from the target site.

- Convection- enhanced delivery (CED) is a type of direct administration that makes the use of positive pressure to increase circulation of drug throughout the tumor.

- CED is practiced either in acute infusions by attaching an implanted catheter to an external pump. For more prolonged infusions, catheters can be attached to implantable osmotic pumps.

- Using CED, high concentrations of drugs can be delivered in tumor tissue. Through careful stereotaxic implantation of CED catheters, normal tissue can be spared. Poor cannula placement can result in leakage of drug away from the target site, potentially leading to adverse effects, such as chemical meningitis.

- The highly variable intracranial distribution of glioma tumors is the additional challenge for the use of CED as it requires an individualized cannula placement plan for each patient. 
- Optimal cannula placement can be aided by the FDA-approved software iPlan by BrainLAB, which models fluid flow in the brain and provides feedback regarding cannula location. iPlan algorithms are rapidly evolving to assist in more accurate cannula placements and more thorough drug distribution.

\section{Protein delivery by CED}

Due to high molecular weight, therapeutic proteins are common candidates for CED. Most therapeutic proteins investigated for the treatment of malignant glioma consist of a cytotoxic moiety, often a bacterial toxin, combined with a targeting moiety for selective therapeutic effect on glioma cells. Cytotoxic moiety is derived from Pseudomonas aeruginosa exotoxin (PE). Other notable fusion proteins being investigated for efficacy by CED include, TransMD- 107 (Tf-CRM107). TransMD-107 is a diphtheria exotoxin fused to Tf and binds to the Tf receptor, which is commonly over expressed in malignant glioma. The safety of TransMD-107 has been demonstrated in Phase I and Phase II studies of clinical trials. CED of oligonucleotides and liposomes: CED is the delivery method of choice for large molecules and biologics, such as oligonucleotides, drug bearing liposomes and viral mediated therapies. Trabedersen (AP-12009) is an antisense oligonucleotide that targets the transcript for Transforming Growth Factor-beta2 (TGF- $\beta 2$ ), a protein involved in tumor growth and survival. Liposomal drug carriers encapsulate small molecule chemotherapeutics and can improve the pharmacokinetic profile of these agents by reducing drug clearance time. When delivered by CED, liposomal agents achieve acceptable tumor coverage, as indicated by MRI using gadolinium-loaded liposomes. Irinotecan has shown impressive efficacy in preclinical studies when delivered by CED. Tumor cells responded with a 15-fold reduction in proliferation. Liposomal Irinotecan decreased tumor vascular density. Though of tremendous potential to improving clinical outcomes for malignant glioma patients, CED faces several challenges for achieving more widespread use Highly variable nature of CED-associated therapeutic distribution in tumors. Procedure is highly invasive III. Limitations in the number of dosing cycles.

\section{Modified nanoparticles}

The utilization of the nanoparticles as a vector for brain drug delivery has the following advantages:

Excellent engineerability

Non-toxicity

Controllable loading/releasing of active agents (drugs/contrast agents)

Targeted nanoparticles can achieve the delivery of large amounts of therapeutic or imaging agents

The nanoparticles with enhanced surface properties (targeting and/or hydrophilic coating) may be able to deliver a high amount of drugs/contrast agents selectively to tumor sites and improve the efficacy of existing imaging and treatment of cancer in general.

The exact mechanism of nanoparticle transport into the brain is most likely

Receptor-mediated endocytosis

Phagocytosis

Passive leakage across defects in the $\mathrm{BBB}^{53}$

\section{CONCLUSION}

The treatment of brain diseases is particularly challenging because the delivery of drug Molecules to the brain is often precluded by a variety of physiological, metabolic and Bio chemical obstacles that collectively comprise the BBB, BCB and BTB. The present outlook for patients suffering from many types of CNS diseases remains poor, but recent developments in drug delivery techniques provide reasonable hope that the formidable barriers shielding the CNS may ultimately be overcome. Drug delivery directly to the brain interstitium has recently been markedly enhanced through the rational design of polymer-based drug delivery systems. Substantial progress will only come about, however, if continued vigorous research efforts to develop more therapeutic and less toxic drug molecules are paralleled by the aggressive pursuit of more effective mechanisms for delivering those drugs to their brain targets.

\section{REFERENCES}

[1].N.K.Jain. Controlled and Novel Drug Delivery, CBS publication, New Delhi, 1st edition reprint 100-130,147- 170, 304-352: 2008.

[2].Pardridge WM. CNS drug design based on principles of blood-brain barrier transport. J Neurochem. 1998; 70: 17811792.

[3].Siegal T, Zylber-Katz E. Strategies for increasing drug delivery to the brain: focus on brain lymphoma. Clin Pharmacokinet. 2002; 41:171-186. 9. Witt KA, Davis TP. CNS drug delivery: Opioid peptides and the blood-brain barrier. The AAPS Journal. 2006; 8: E76- E88.

[4].trotta M, caputo O, Debernardi F. Preparation of solid lipid nanoparticals by a solvent emulsification-diffusion technique. International Journal of pharmacy 2003; 257: 153-60. http://dx.doi.org/10.1016/S03785173(03)00135-2

[5].Drug delivery to the central nervous system: a review, Ambikanandan Mishra, Ganesh S., Aliasgar Shahiwal, Shrenik P. Shah, Received 16June 2003.

[6].www.slideshare.net 25-06-2015. 5. Dm Brahmankar,Sunil B Jaiswal. Biopharmaceutics \& Pharmacokinetics A Treatise, Vallabh Publications 2nd edition reprint 397-429, 495-501:2004.

[7].N.K.Jain. Controlled and Novel Drug Delivery, CBS publication, New delhi, 1st edition reprint 100-130,147- 170, 304-352: 2008.

[8].K. A. Witt, T. J. Gillespie, J. D. Huber, R. D. Egleton, T. P. Davis, Peptides 2001, 22, 2329. 
[9].M. S. Alavijeh, M. Chishty, M. Z. Qaiser, A. M. Palmer, NeuroRx 2005, 2, 554.

[10]. R. D. Egleton, T. P. Davis, Peptides 1997, 18, 1431.

[11]. J. F. Deeken, W. Loscher, Clin. Cancer Res. 2007, 13, 1663.

[12]. W. M. Pardridge, Pharm. Sci. Technol. Today 1999, 2, 49.

[13]. A. G. de Boer, P. J. Gaillard, Clin. Pharmacokinet. 2007, 46, 553.

[14]. Siegal, T. and zylber-Katz, E., Strategies for increasing drug delivery to the brain: focus on brain lymphoma, Clin Pharmacokinet, 41:171-186, 2002.

[15]. Arun Rasheed, I Theja, et al., CNS TARGETED DRUG DELIVERY: CURRENT PERSPECTIVES, JITPS 2010 , Vol. 1 (1), 9-18.

[16]. Gabathuler R. Approaches to transport therapeutic drugs across the blood-brain barrier to treat brain diseases. Neurobiol. Dis. 37(1), 48-57 (2010).

[17]. Jones AR, Shusta EV. Blood-brain barrier transport of therapeutics via receptor- mediation. Pharm. Res. 24(9), 1759-1771 (2007).

[18]. Pardridge WM. Brain drug development and brain drug targeting. Pharm. Res. 24(9), 1729- 1732 (2007).

[19]. Pardridge WM. Drug targeting to the brain. Pharm. Res. 24(9), 1733-1744 (2007).

[20]. Rip J, Schenk GJ, de Boer AG. Differential receptor-mediated drug targeting to the diseased brain. Expert Opin. Drug Deliv. 6(3), 227-237 (2009).

[21]. Bickel U, Yoshikawa T, Pardridge WM. Delivery of peptides and proteins through the blood-brain barrier. Adv. Drug Deliv. Rev. 46(1-3), 247-279 (2001).I

[22]. Lu W, Wan J, She Z, Jiang X. Brain delivery property and accelerated blood clearance of cationic albumin conjugated pegylated nanoparticle. J. Control. Release 118(1), 38- 53 (2007).

[23]. Reddy JS, Venkateswarlu V. Novel delivery systems for drug targeting to the brain. Drugs Future 2004; 29 : 63-69.

[24]. Kabanov AV, Batrakova EV. New technologies for drug delivery across the blood brain barrier. Curr Pharm Des 2004; 10: 1355-1363.

[25]. Mishra A, Ganesh S, Shahiwala A, Shah SP. Drug delivery to the central nervous system: a review. J Pharm Pharm Sci 2003; 6(2): 252-273.

[26]. Huwyler J, Wu D, Pardridge WM. Brain drug delivery of small molecules using immunoliposomes. Proc Natl Acad Sci USA 1996; 93: 14164-14169.

[27]. Pardrige, W.M., Huwyler, J.: WO022092A1 (1998).

[28]. Tosi, G.; Costantino, L.; Ruozi, B.; Forni, F.; Randelli, M.A. Polymeric nanoparticles for the drug delivery to the central nervous system. Exp. Opin. Drug Deliv. 2008, 5 (2), 155-174.

[29]. Vyas SP, Khar RK. Targeted and controlled drug delivery. 1st ed. Chennai (India): CBS Publishers and distributers Pvt. Ltd.; 2002.

[30]. Moghimi, S.M.; Hunter, A.C.; Murray, J.C. Long-circulating and target-specific nanoparticles: theory to practice. Pharmacol. Rev. 2001, 53, 283-318.

[31]. Ogawara, K.; Furumoto, K.; Takakura, Y.; Hashida, M.; Higaki, K.; Kimura, T. Surface hydrophobicity of particles is not necessarily the most important determinant in their in vivo disposition after intravenous administration in rats. J. Control. Release 2001, 77, 191-198.

[32]. Huwyler J, Wu D, Pardridge WM. Brain drug delivery of small molecules using immunoliposomes. Proc Natl Acad Sci USA 1996; 93: 14164-14169.

[33]. Pardrige, W.M., Huwyler, J.: WO022092A1 (1998).

[34]. Tosi, G.; Costantino, L.; Ruozi, B.; Forni, F.; Randelli, M.A. Polymeric nanoparticles for the drug delivery to the central nervous system. Exp. Opin. Drug Deliv. 2008, 5 (2), 155-174.

[35]. Yang, S.; Zhu, J.; Lu, Y.; Liang, B.; Yang, C. Body distribution of camptothecin solid lipid nanoparticles after oral administration. Pharm. Res. 1999, 16, 751-757.

[36]. Zara, G.P.; Cavalli, R.; Bargoni, A.; Fundaro, A.; Vighetto, D.; Gasco, M.R. Intravenous administration to rabbits of non-stealth and stealth doxorubicin-loaded solid lipid nanoparticles at increasing concentrations of stealth agent: pharmacokinetics and distribution of doxorubicin in brain and other tissues. J. Drug Target. 2002, 10, $327-335$.

[37]. Wong, H. L.; Bendayan, R.; Rauth, A. M.; Li, Y.; Wu, X.Y. Chemotherapy with anticancer drugs encapsulated in solid lipid nanoparticles. Adv. Drug Deliv. Rev. 2007, 59, 491-504

[38]. Chattopadhyay, N.; Zastre, J.; Wong, H.L.; Wu, X.Y.; Bendayan, R. Solid lipid nanoparticles enhance the delivery of the HIV protease inhibitor, atazanavir, by a human brain endothelial cell line. Pharm. Res. 2008, 25, $2262-71$.

[39]. Adams ML, Lavasanifar A, Kwon GS. Amphiphilic block copolymers for drug delivery. J Pharm Sci 2003; 92 : 1343-1355.

[40]. Jones M, Leroux J. Polymeric micelles-a new generation of colloidal drug carriers. Eur J Pharm Biopharm 1999; 48: 101-111.

[41]. Allen C, Maysinger D, Eisenberg A. Nano- engineering block copolymer aggregates for drug delivery. Colloids Surf B Biointerfaces 1999; 16: 3-27.

[42]. Kabanov AV, Batrakova EV, Melik-Nubarov NS, et al. New classes of drug carries: micelles of poly(oxyethylene) - poly(oxypropylene block copolymersas microcontainers for drug targeting form blood in brain. J Control Release 1992; 22: 141-158.

[43]. Huwyler J, Wu D, Pardridge WM. Brain drug delivery of smallmolecules using immunoliposomes. Proc Natl Acad Sci USA 1996; 93: 14164-14169. 
[44]. C. Rousselle, P. Clair, J. M. Lefauconnier, M. Kaczorek, J. M. Scherrmann, J. Temsamani, Mol. Pharmacol. 2000, 57,679 .

[45]. Alam MI, Beg S, Samad A, Baboota S, Kohlia K, Ali J, Ahuja A, Akbar M. Strategy for effective brain drug delivery: Eur J Pharm Sci 2010; 40:385-403.

[46]. Jain JP, Modia S, Dombb AJ, Kumar N. Role of polyanhydrides as localized drug carriers: J Controlled Release 2005; 103:541- 563.

[47]. Moses MA, Brem H, Langer, R. Advancing the field of drug delivery: Taking aim at cancer: Cancer cell $2003 ; 4$ Suppl 5:337-341.

[48]. Walter KA, Cahan MA, Gur A, Tyler B, Hilton J, Colvin OM, Burger PC, Domb A, Brem H. Interstitial Taxol delivered from a biodegradable polymer implant against experimental malignant glioma: Cancer Res 1994; 54 Suppl $8: 2207-2212$.

[49]. Gene therapy net.com. http://www.genetherapynet.com/non- viral-vectors/lipoplexes-andpolyplexes.html.

[50]. Sawant RR, Sriraman SK, Navarro G, Biswas S, Dalvi RA, Torchilin, VP. Polyethyleneimine-lipid conjugate-based pH- sensitive micellar carrier for gene delivery: Biomaterials 2012; 33 Suppl 15:3942-51.

[51]. Zanta MA, Belguise-Valladier P, Behr JP. Gene delivery: A single nuclear localization signal peptide is sufficient to carry DNA to the cell nucleus: Proc Natl Acad Sci USA 1999; 96:91-96.

[52]. Woerly S, Petrov P, Sykova E, Roitbak T, Simonova Z, Harvey AR. Neural tissue formation within porous hydrogels implanted in brain and spinal cord lesions: Ultrastructural, immunohistochemical and diffusion studies: Tissue Eng 1999; 5:467-488.

[53]. Serwer LP, James CD. Challenges in drug delivery to tumors of the central nervous system: An overview of pharmacological and surgical considerations: Adv Drug Deli Rev 2012; 64 Suppl 7:590-597. 